\title{
A review of tooth discoloration and staining
}

\author{
Tooth discolouration and staining: a review of the literature by A. Watts and M. Addy Br Dent J 2001; 190: \\ 309-316
}

\author{
Objective \\ To carry out an extensive review of the literature on tooth-staining \\ with particular regard to some of the more recent literature on \\ the mechanisms of tooth-staining involving mouthrinses.

\section{Design} \\ Comprehensive review of the literature over four decades.

\section{Conclusions} \\ A knowledge of the aetiology of tooth-staining is of importance \\ to dental surgeons in order to enable a correct diagnosis to be \\ made when examining a discoloured dentition and allows the \\ dental practitioner to explain to the patient the exact nature of \\ the condition. In some instances, the mechanism of staining may \\ have an effect on the outcome of treatment and influence the \\ treatment options the dentist will be able to offer \\ to patients.
}

\author{
In Brief \\ - Patients increasingly attend dental surgeons for dental work \\ to improve their appearance \\ - Discolouration of the teeth and staining has an effect on \\ appearance \\ - Some causes of discolouration and staining are more \\ amenable to dental treatment \\ - Ability to recognise and diagnose the cause of \\ discolouration and staining may help treatment planning
}

\section{Comment}

lthough the discolouration and stainAing of teeth is of considerable interest to our patients, primarily for aesthetic reasons, it is not a subject area which has received a great deal of attention in the scientific literature. Perhaps surprisingly standard texts on oral and dental diseases in the middle of the last century often included a whole chapter on this particular topic. However, it has since been relegated to a few paragraphs in more modem texts on oral medicine or oral pathology.

This review is therefore a timely reminder to the profession that, whilst as dentists we may regard tooth staining as relatively unimportant, the subject looms large in the questions put to us by our patients. The literature on the subject is widely dispersed and a review bringing together information currently available is to be commended.

Watts and Addy in this journal present such a review, being the only one readily available since 1975 . Their stated objective is to enable dental practitioners to understand the causes of the condition in order to make a diagnosis and to enable appropriate treatment to be carried out.
The review sets the scene by describing the mechanisms of colour perception and the problems of inaccuracy under the conditions of lighting frequently found in the dental surgery. Their classification of stains is simple in that intrinsic tooth discolouration is distinguished from extrinsic staining, although the latter is complicated by what the authors refer to as internalised discolouration which, in effect, means the take up of extrinsic stains by the tissues and tooth.

Within these broad headings there is an extensive review of pathological conditions resulting in intrinsic changes of tooth colour. There is little to add to their comments, although under the heading of 'root resorption' it is not immediately clear that they are referring to internal resorption of the root resulting in visibility of the pink tooth tissue through the tooth substance.

Their brief comment on the changing colour of teeth as age increases should perhaps have mentioned that workers in the field of forensic dentistry have used this phenomenon as a means of age determination at death, not only in human beings but interestingly also in baboons.
It is to be expected from these authors that the relationship of staining to the use of such antiseptics as chlorhexidine would be extensively dealt with and the reader may permit her/himself a smile at the thought of red wine and tea drinking rabbits.

The review is a comprehensive and useful addition to the literature but the stated objective of enabling ready diagnosis and treatment planning to be made will perhaps require a further communication. In relation to the former aim it would perhaps be useful to have the various stains listed in a table in terms of their colour or hue, coupled with the conditions related to this phenomenon. The practitioner is left waiting for any comment regarding the correct treatment for each of the conditions listed.

To conclude, this extensive review is timely and comprehensive and will bring us all up to date in relation to this condition of such importance in these days of cosmetic dentistry.

\section{Professor David Whittaker}

Professor of Forensic Dentistry, The Dental

School, University of Wales College of

Medicine, Cardiff 